\title{
Extracellular vesicle (EV)-polyphenol nanoaggregates for microRNA-based cancer diagnosis
}

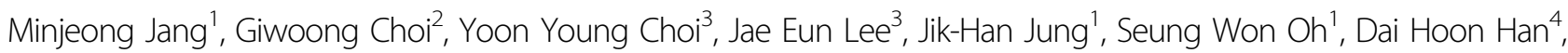 \\ Haeshin Lee ${ }^{5}$, Ji-Ho Park', Jae-Ho Cheong ${ }^{3}$ and Pilnam Kim,
}

\begin{abstract}
Small extracellular vesicles (EVs), including exosomes, in body fluids have important applications in the noninvasive liquid biopsy-based diagnosis of cancer. Current EV-based diagnostic techniques still face practical challenges, such as inefficient EV isolation. Here, we report an efficient, resource-free pre-enrichment approach using (-)-epigallocatechin3-gallate (EGCG), a polyphenolic biomolecule, to isolate and detect exosomal microRNAs (miRNAs) in human blood plasma samples. Our system comprises three steps: (1) EGCG-mediated EV aggregation, (2) filter-based EV isolation, and (3) molecular beacon-based detection of target miRNA in EVs. Using blood samples from cancer patients with gastric cancer or hepatocellular carcinoma, we constructed an EGCG-assisted miRNA diagnostic system. For both cancers, the levels of target miRNAs (miR-21,-27a, and -375) in EVs were strongly correlated with those in the publicly available GEO database. Our approach, an easy-to-use method for efficient EV isolation and the detection of miRNA in clinical samples, is applicable for molecular diagnostics in precision medicine.
\end{abstract}

\section{Introduction}

Extracellular vesicles (EVs) are spherical bilayered phospholipid vesicles released from cells that contain various biocomponents, such as RNA, DNA, proteins, and lipids ${ }^{1-4}$. Consequently, EVs can provide comprehensive information about a particular cancer, such as genetic alterations and gene expression patterns ${ }^{1}$. Several studies have suggested that tumor-derived exosomes are important cancer markers that can be serially detected with minimal invasion ${ }^{5,6}$. Although they have been significantly improved by micro- and nanoengineering strategies ${ }^{7-17}$, exosome-based diagnostics still struggle to meet the demands of clinical applications involving point-of-care testing $(\mathrm{PoCT})^{18}$, which requires a resource-free

\footnotetext{
Correspondence: Pilnam Kim (pkim@kaist.ac.kr)

${ }^{1}$ Department of Bio and Brain Engineering, Korea Advanced Institute of Science and Technology (KAIST), Daejeon 34141, South Korea

2Department of Mechanical Engineering, Korea Advanced Institute of Science and Technology (KAIST), Daejeon 34141, South Korea

Full list of author information is available at the end of the article
}

approach, ease of use, low cost, reliability, and scalability. In particular, the clinical utility of exosomes has been limited by technical obstacles associated with their physical properties (i.e., their small size of $<100 \mathrm{~nm}$ ) and the extensive sample preparation required prior to measurement due to their extremely low concentration $\left(<10^{9}\right.$ vesicles $/ \mathrm{mL}$ of serum $)^{7}$. Thus, there is a clear need to modify the existing method of exosome utilization in clinical diagnostics.

Polyphenolic biomolecules, including (-)-epigallocatechin-3-gallate (EGCG), contain many catechol and gallol functional groups that influence protein binding and aggregation via hydrophobic interactions, hydrogen bonding, and $\pi-\pi$ stacking interactions ${ }^{19-24}$. The strong adhesive properties of polyphenol to biological molecules ${ }^{25}$ permit the collection of exosomes in biofluids (e.g., whole blood, plasma, serum, and urine) for diagnostic and clinical assessment. A recent study demonstrated that green tea-derived polyphenolic molecules interact with isotropic bicelle lipid membranes; likewise, the gallol ring

\section{(c) The Author(s) 2019}

(c) (i) Open Access This article is licensed under a Creative Commons Attribution 4.0 International License, which permits use, sharing, adaptation, distribution and reproduction cc) in any medium or format, as long as you give appropriate credit to the original author(s) and the source, provide a link to the Creative Commons license, and indicate if changes were made. The images or other third party material in this article are included in the article's Creative Commons license, unless indicated otherwise in a credit line to the material. If material is not included in the article's Creative Commons license and your intended use is not permitted by statutory regulation or exceeds the permitted use, you will need to obtain permission directly from the copyright holder. To view a copy of this license, visit http://creativecommons.org/licenses/by/4.0/. 
of EGCG interacts with the trimethylammonium group on the surface of lipid membranes ${ }^{21,24,26}$. Based on this mechanism of polyphenol-phospholipid binding ${ }^{21,24,26}$, we speculated that polyphenolic molecules, including EGCG, can be used as part of an exosome isolation/preenrichment strategy for liquid biopsy-based diagnosis.

In this study, we designed efficient pre-enrichment and isolation methods for exosomal miRNA-based cancer diagnosis. To achieve this objective, we applied a sequential procedure involving the enrichment of exosomes through adhesion to EGCG (Fig. 1a) and the collection of exosome aggregates from the EGCG/biofluid mixture by manual filtration. Nanosized exosomes in biofluids, including conditioned media (CM) and human blood plasma samples, formed a few hundred nanosized aggregates via a robust interaction between EGCG and exosomes (Fig. 1b). Bundles of aggregated exosomes were efficiently isolated by filtration, and multiplexed exosomal miRNA was analyzed using a molecular beacon (MB) hybridization method for the detection and quantification of cancer biomarkers. We then analyzed and compared the miRNA expression results using real-time reverse transcription-polymerase chain reaction (RT-qPCR).

\section{Materials and methods}

\section{Gastric cancer (GC) cell culture and conditioned medium} collection

The AGS, MKN-74, and Hs746T human GC cell lines were cultured in RPMI 1640 (WelGENE, Korea) supplemented with $10 \%$ fetal bovine serum (FBS; WelGENE) and $1 \%$ penicillin/streptomycin (P/S; WelGENE). SKGT-4 cells, a human esophageal adenocarcinoma cell line, were also cultured in RPMI 1640 supplemented with 10\% FBS and $1 \% \mathrm{P} / \mathrm{S}$. HEK293T cells and human fibroblasts (hTERT fibroblasts) were used as representative normal human cells and cultured in Dulbecco's modified Eagle's medium (DMEM) and DMEM/F12 (WelGENE), respectively, supplemented with $10 \% \mathrm{FBS}$ and $1 \% \mathrm{P} / \mathrm{S}$. The supplemented media were considered complete media. Cells were incubated in a humidified incubator at $37^{\circ} \mathrm{C}$ with $5 \% \mathrm{CO}_{2}$. To obtain exosomes, cells were cultured in complete medium to $70-80 \%$ confluency and then cultured in FBS-free medium for an additional $48 \mathrm{~h}$ ( $24 \mathrm{~h}$ for HEK293T cells) to minimize the levels of unwanted FBSassociated exosomes. After $48 \mathrm{~h}$ ( $24 \mathrm{~h}$ for HEK293T cells), the medium was collected and filtered using 450-nm syringe filters (Pall Life Sciences, USA) to remove cellular debris and stored at $4{ }^{\circ} \mathrm{C}$; this medium was considered conditioned medium (CM).

\section{Preparation of blood plasma samples from cancer patients and healthy donors}

Plasma samples from GC and hepatocellular carcinoma (HCC) patients and healthy donors were collected following Institutional Review Board (IRB) guidelines. This study was approved by the IRBs of Yonsei University Severance Hospital (4-2017-0379) and the IRB of the Korea Advanced Institute of Science and Technology (KAIST; KH2018-53). Plasma samples were obtained from three GC patients, three HCC patients, and five healthy donors. Patients expected to undergo gastrectomy or liver resection due to $\mathrm{GC}$ or $\mathrm{HCC}$, respectively, at Yonsei University Severance Hospital were eligible for this study. Five milliliters of each patient's whole blood was taken a day before or on the day of surgical operation and placed in an EDTA tube. At least $500 \mu \mathrm{L}$ of blood plasma was acquired by the centrifugation of whole blood from healthy donors and patients. Plasma samples were stored at $-80^{\circ} \mathrm{C}$ until analysis.

\section{Green tea extract-based exosome isolation}

Exosomes from plasma and the CM of a variety of other cell lines were isolated using an solution of $1 \mathrm{mg} / \mathrm{mL}$ EGCG (Sigma, USA) in ultrapure water (WelGENE). Plasma and CM were each combined with the EGCG solution at a $3: 1$ ratio, kept at $4{ }^{\circ} \mathrm{C}$ overnight, and centrifuged at $3000 \mathrm{rpm}$ for $20 \mathrm{~min}$ at $4{ }^{\circ} \mathrm{C}$.

\section{Filter-based EV isolation}

To easily isolate exosome aggregates, we used a resourcefree and reusable plastic filter holder (Pall Life Sciences) and membrane filters (Supor-450; Pall Life Sciences); the filter holder and membrane filter were tightly assembled with a rubber O-ring. EGCG-treated CM and plasma samples were filtered through the assembled filter holder and washed twice with Dulbecco's phosphate-buffered saline (DPBS). Various volumes of CM $(0-10 \mathrm{~mL})$ and plasma samples $(500 \mu \mathrm{L})$ diluted 10-fold in DPBS were used. The filter holder was reused after autoclaving, and the membrane filter was used for subsequent work, including exosomal miRNA detection by RT-qPCR and MB.

\section{Analysis of size distribution}

The size distributions of the exosome aggregates in CM with and without EGCG treatment were determined using a Zetasizer instrument (Malvern, UK) in triplicate.

\section{EV imaging by electron microscopy}

The microstructure of the isolated exosome aggregates was imaged using field emission scanning electron microscopy (FE-SEM) and field emission-transmission electron microscopy (FE-TEM). In preparation for FESEM imaging, isolated exosome aggregates were fixed with $2.5 \%(\mathrm{v} / \mathrm{v})$ glutaraldehyde (Sigma) for $2 \mathrm{~h}$; the fixed samples were then dehydrated with serially diluted ethanol (30, 50, 70, 90, 95, 100, and 100\%) for 15 min each. The dehydrated samples were frozen, vacuum-dried overnight, and observed directly by FE-SEM (Hitachi, 


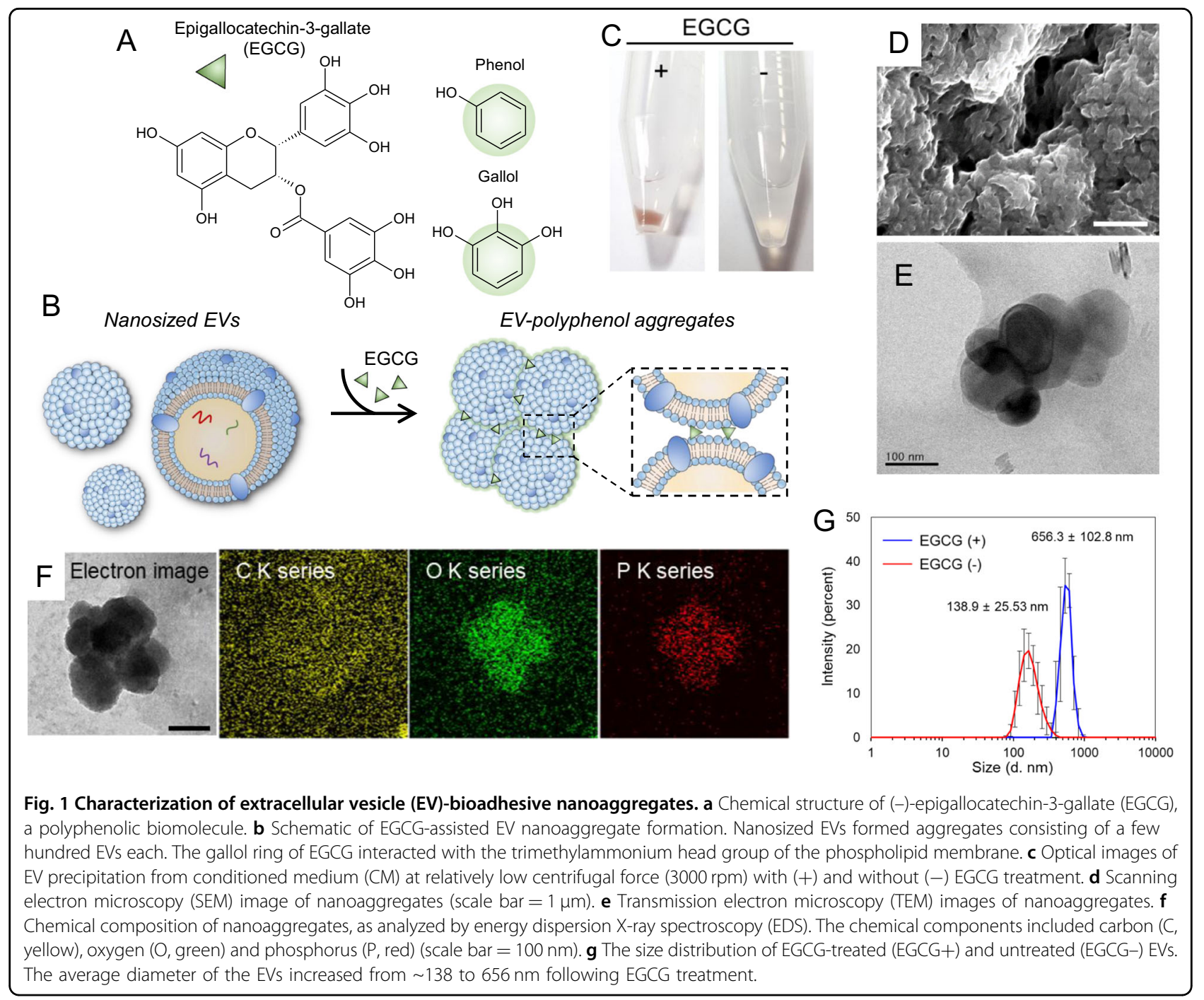

Japan) after the application of a thin Pt coating. In preparation for FE-TEM imaging, exosome pellets were resuspended in DPBS twice. We then added $10 \mu \mathrm{L}$ of the exosome-containing DPBS solution to a Formvar/copper grid (Electron Microscopy Sciences, USA), and any excess solution after $1 \mathrm{~min}$ was removed by absorption with filter paper. Exosomes on the Formvar/copper grid were then negatively stained with a $1 \%$ phosphotungstic acid solution ( $\mathrm{pH} 7.5$, neutral; Sigma) for $30 \mathrm{~s}$ and analyzed by FETEM (JEM-2100F; JEOL, Japan) with an accelerating voltage of $200 \mathrm{kV}$. To confirm the chemical components in the exosome aggregates, energy-dispersive $\mathrm{X}$-ray spectroscopy (EDS) was performed conventionally using the $\mathrm{K}$ series with TEM.

\section{Synthetic miRNA detection using MBs}

Fluorescently labeled MBs and synthetic miRNAs were synthesized by Macrogen (Korea). The MBs were designed to target miR-21, miR-27a, and miR-375. Supplementary Table S2 shows the sequences of the MBs and synthetic miRNAs. Synthetic miRNA has a similar sequence to that of natural mature miRNA, except the uracil in natural miRNA is replaced by thymidine.

\section{Detection of exosomal miRNA using MBs}

We directly introduced lysis solution (a $1 \%$ Triton X100 solution in DPBS) to the centrifuged EV aggregate pellet, followed by lysis and hybridization with MBs. In the case of the filtered EVs, lysis solution with MBs was directly introduced to the filter membrane in a $1.5 \mathrm{~mL}$ microtube $(1.5 \mathrm{~mL}$ TubeOne microcentrifuge tubes, Starlab). To detect single exosomal miRNAs, isolated exosomes were incubated with $200 \mathrm{nM} \mathrm{MB}$ in lysis solution (a 1\% Triton X-100 solution in DPBS) for $30 \mathrm{~min}$ at room temperature (RT). To detect multiplexed exosomal miRNA, $200 \mathrm{nM}$ MBs in lysis solution (a 1\% Triton X-100 
solution in DPBS) were incubated together for $30 \mathrm{~min}$ at RT. The signal-to-background (S/B) ratio was measured using a multimode microplate reader (SpectraMax ID3; Molecular Devices, USA). The background signal was the fluorescence intensity from the MB without exosomes.

\section{Total RNA extraction and RT-qPCR analysis of exosome aggregates}

Total RNA was isolated from exosome aggregates using TRIzol RNA lysis reagent (Ambion, USA) by the phenol/ chloroform method. Briefly, TRIzol reagent was directly introduced to centrifuged EV pellets and filtered EVs on the filter membrane, followed by phase separation using chloroform (Sigma). After separating the transparent RNA-containing phase, RNA was precipitated by treatment with isopropyl alcohol and glycogen for $1 \mathrm{~h}$ at $-20^{\circ} \mathrm{C}$. The precipitated RNA was then collected and washed with 70\% ethanol. Dried RNA was dissolved in a suitable amount of diethylpyrocarbonate (DEPC) water (WelGENE). The total RNA concentration was determined by measuring the absorbance at $260 / 280 \mathrm{~nm}$ using a SpectraDrop Micro-volume microplate reader (Molecular Devices, USA).

\section{RT-qPCR assay}

RT-qPCR was performed to analyze the exosomal miRNA in CM and human blood plasma. The extracted RNA samples were reverse-transcribed to cDNA with $5 \times$ HiSpec buffer using the miScript RT II kit (Qiagen, Germany). Primers for miR-21, -27a, -375, and RNU6B were purchased from Qiagen. Target miRNAs were normalized to RNU6B miRNA, which was used as a reference gene. RT-PCR to amplify mature miR-21, $-27 \mathrm{a}$, and -375 was performed using the miScript SYBR green PCR kit (Qiagen) and measured using the CFX Real-Time system (Bio-Rad, USA).

\section{miRNA expression profile analysis based on the GEO database}

We obtained information from the online-accessible GEO database for a comparative analysis of miR-21, -27a, and -375 levels in GC, HCC, and normal cells. We selected GEO numbers GSE23739 and GSE54397 from the miRNA database as representative GC cell data and GEO numbers GSE21362 and GSE22059 as representative HCC cell data.

\section{Comparison of EV isolation efficiency and purity with commercial kits and EGCG}

To compare the efficiency and purity of the isolated EVs, exosome surface markers (CD9 and CD63) and Apo $\mathrm{B}$ were targeted. We analyzed EVs from conditioned media and patient plasma. When using patient plasma, we combined 5 samples at equal volumes because of the small sample volume. Briefly, exosomal proteins in exosomes precipitated by using EGCG and commercial kits (\#1 and \#2) were extracted in $1 \times$ RIPA buffer (Sigma) supplemented with Halt protease inhibitor cocktail (Invitrogen, USA). The concentration of the extracted proteins was measured by the Bradford assay (Bio-Rad). Extracts containing equal amounts of protein were loaded onto 15\% SDS-PAGE gels and transferred to nitrocellulose membranes (Amersham, UK). Anti-CD9, anti-CD63, and anti-Apo B antibodies were purchased from Santa Cruz Biotechnology (USA). Members were incubated with primary antibodies at $4{ }^{\circ} \mathrm{C}$ overnight, and HRPconjugated secondary antibodies were added for $1 \mathrm{~h}$ at RT. The level of albumin, the most abundant plasma protein, was analyzed by Ponceau S (Sigma) staining. Band signals were detected by using ECL Western blotting detection reagent (Invitrogen), and the membranes were imaged using a membrane imaging system (ImageQuant LAS 4000 mini, GE Healthcare, USA).

\section{Statistical analysis}

Normalized values from independent experiments are expressed as the average \pm standard deviation (SD). Significance was statistically analyzed using two-tailed Student's $t$-tests, and $p$-values are denoted by asterisks $\left({ }^{*}\right)$ as follows: $" p<0.05, * * p<0.01, * * * p<0.001$, and $* * * * * p<$ 0.0001 . All experiments were conducted independently in triplicate.

\section{Results}

\section{Characterization of isolated EVs}

We observed that nanosized EV in biofluid formed EV aggregate bundles through the adhesive properties of EGCG (Fig. 1b). Unlike EV aggregate precipitation using ultracentrifugation (up to $1,000,000 \times g$ ), the gold standard method for EV extraction ${ }^{27}$, we were able to easily precipitate EV aggregate bundles using a much lower centrifugal force $(3000 \mathrm{rpm})$ (Fig. 1c). To determine the optimal isolation conditions, we measured the total RNA concentration based on EGCG concentration and processing time (Fig. S1). Optimal isolation conditions to ensure better performance with high concentrations of extracted total RNA involved the use of a $1 \mathrm{mg} / \mathrm{mL}$ EGCG solution (Fig. S1A) over a $10-12 \mathrm{~h}$ processing time (Fig. S1B). The other polyphenolic biomolecules catechin (1 mg/mL in $50 \%$ ethyl alcohol), gallic acid (GA, $1 \mathrm{mg} / \mathrm{mL}$ in $50 \%$ ethyl alcohol), tannic acid (TA, $1 \mathrm{mg} / \mathrm{mL}$ in distilled water), and naringenin $(1 \mathrm{mg} / \mathrm{mL}$ in $50 \%$ ethyl alcohol) formed precipitates containing EVs in CM at a higher rate than DPBS, which was used as a negative control (Fig. S2).

Upon examining the morphology of EV-bioadhesive nanoaggregates using FE-SEM and HR-TEM (Fig. 2d, e), we observed precipitated aggregate bundles that were 
$100-200 \mathrm{~nm}$ in size. The EVs were round in shape with a thin phospholipid bilayer approximately $5 \mathrm{~nm}$ in thickness. We used EDS to analyze the chemical EV composition in detail, and chemical mapping showed that the EV-bioadhesive nanoaggregates contained phosphorus (red) in their phospholipid bilayer (Fig. 2f). The average diameter of the nanoaggregates following treatment with EGCG (EGCG $(+)$ ) was $656.3 \pm 102.8 \mathrm{~nm}$, while that of nanoaggregates in CM not treated with EGCG was $138.9 \pm 25.53 \mathrm{~nm}$ (EGCG (-), Fig. 2g), indicating that EGCG treatment increased the overall nanoaggregate size.

\section{Comparison of EV purity and isolation efficiency with polyphenol-assisted isolation to those with isolation via commercial kits}

Polyphenol-assisted EV isolation was compared with EV isolation using reagents from commercial kits (CKs): ExoQuick Exosome Precipitation Solution (System Biosciences, CK \#1) and Total Exosome Isolation Reagent (Invitrogen, CK \#2). Supplementary Table S1 briefly describes the CKs. Following the manufacturer's instructions, we mixed $10 \mathrm{~mL}$ of $\mathrm{CM}$ with $\mathrm{CK} \# 1$, CK \#2, and EGCG for comparison; EVs were then isolated by centrifugation with the appropriate centrifugal force recommended for the CKs (CK \#1: 3,000 rpm, CK \#2: $10,000 \mathrm{rpm}$ ) or our filter-based method in the case of EGCG treatment. We observed differences in the quantity of centrifuge-precipitated EVs (Fig. 2a) and confirmed that the visibly significant increase in precipitation corresponded to increased levels of the exosome surface markers CD9 and CD63 (Fig. 2b) upon analysis. These results indicate that EGCG is more reactive with the biomolecules in $\mathrm{CM}$ than the polymers used in commercialization kits are, resulting in increased precipitation. In comparison, CK \#1 had a better EV isolation efficiency than CK \#2, and EGCG had the highest overall isolation efficiency.

To evaluate isolated EV yields, we compared the concentration of total proteins obtained using our method to that obtained using CK \#1 through the Bradford assay (Fig. S3A). Our EGCG-based method of isolation from $\mathrm{CM}$ and blood plasma yielded 9-fold and 10-fold higher total protein concentrations, respectively, compared to the CK \#1 method (Fig. S3B). Moreover, we evaluated CD63, CD9, Apo B, and albumin levels to assess the purity and recovery of isolated EVs; CD63 and CD9 were used as EV markers, and Apo B was used as a lipoprotein marker. Albumin was targeted because it is the most abundant protein in plasma. EVs were recovered by the CK \#1 method, reflected by detection of the EV markers CD9 and CD63 (Fig. 2c). However, lipoprotein markers (Apo B) and albumin were also detected by Ponceau $\mathrm{S}$ staining (70 kDa, albumin) (Fig. 2d). We confirmed that our method enabled more efficient EV recovery with less contamination from non-EV components than the CK \#1
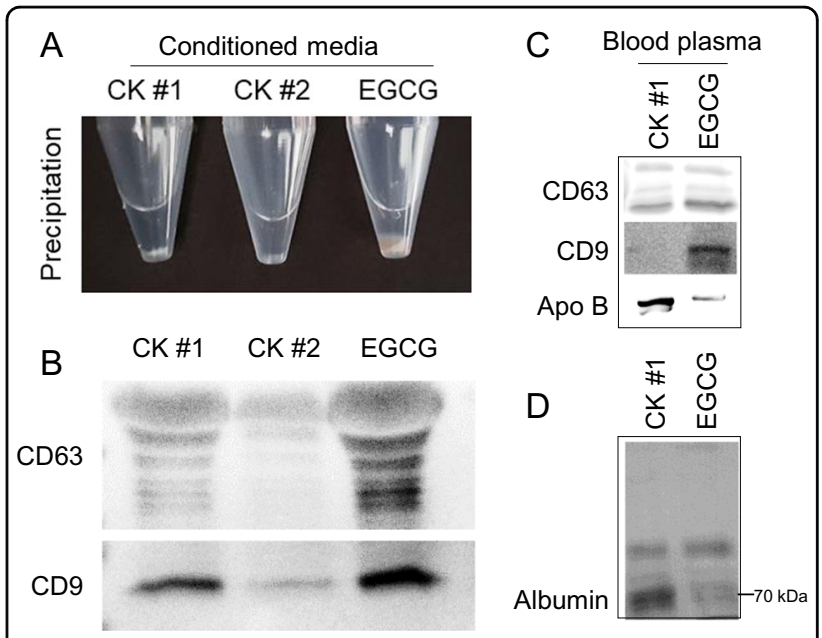

Fig. 2 Comparison of the EV isolation efficiency and EV purity between commercial kit (CK) \#1, CK \#2 and EGCG. a EV precipitation from CM by CKs and EGCG. $\mathbf{b}$ Expression of the exosome surface markers CD9 and CD63 in CM. c, d Expression of CD63, CD9, Apo $B$, and albumin in EVs isolated from blood plasma from patient samples using CK \#1 and EGCG. c Protein bands of CD63, CD9, and Apo $B$ analyzed by Western blotting, with $\mathbf{d}$ the albumin band at $70 \mathrm{kDa}$ observed by Ponceau S staining.

method. The band signal intensities for the EV markers CD63 and CD9 were higher in EGCG-treated blood plasma (by 1.3-fold and 9.9-fold, respectively) compared to CK \#1-treated blood plasma. In contrast, the levels of non-EV markers in EGCG-treated blood plasma were 1.6fold (Apo B) and 4.9-fold (Albumin) lower than those in CK \#1-treated blood plasma (Fig. S3C). Among the various EV isolation methods, commercial EV precipitation kits are used mainly because CKs are easy and simple to use, albeit expensive. Commercial kits use polymer-based precipitation to form a mesh-like net around EVs that range in size from 60 to $180 \mathrm{~nm} ;{ }^{28}$ these wrapped EVs are low in purity. This study indicates that the EGCG-assisted method is more efficient than commercial EV isolation kits and isolates EVs at higher purity. Therefore, the use of EGCG and its derivatives is an economical and more efficient alternative to commonly used but expensive commercial products.

\section{Filter-based EV isolation}

We then performed filter-based EV isolation and collection, which we called the exofilter procedure (Fig. 3a). Based on the results of size analysis (Fig. 1g), we used a membrane filter with a pore size of $450 \mathrm{~nm}$ to collect EVbioadhesive nanoaggregates. For the easy separation and further analysis of filtered EVs, we used an assemblable filter holder as a cartridge for the syringe filter. Figure $3 \mathrm{~b}$ shows SEM images of filtered EV-bioadhesive nanoaggregates in EGCG-treated (EGCG+) CM. EGCG-treated medium was precipitated again after filtration (Fig. 3c) to 


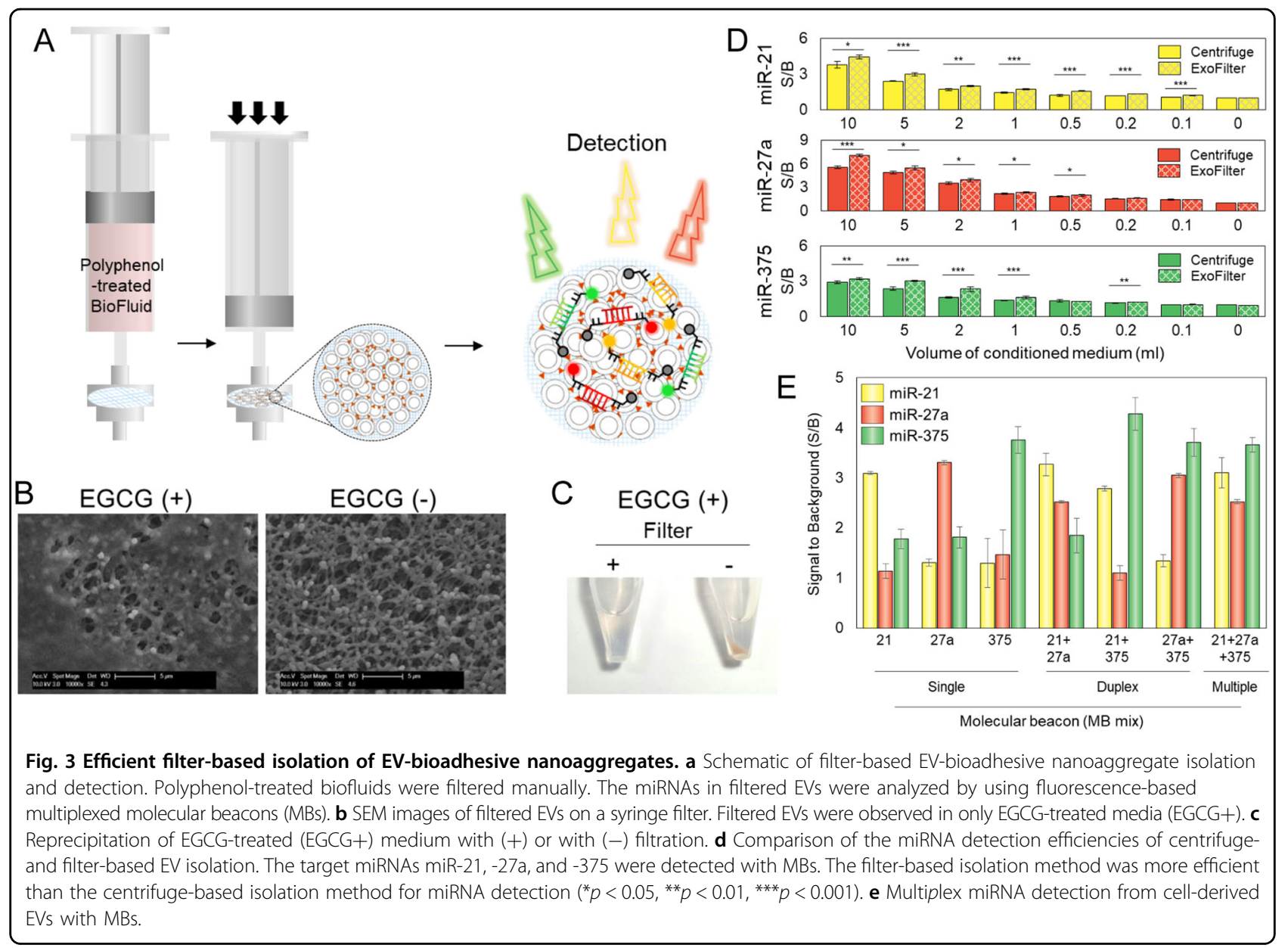

verify the efficiency of filter-based isolation. Filter-based EV isolation appeared to be efficient because no reprecipitation was observed in the EGCG $(+)$ filter $(+)$ CM sample, unlike the filter (-) CM sample.

\section{Molecular beacon-based direct detection of miRNA in EVs}

We performed molecular beacon (MB)-based multiplexed miRNA detection, a simplified detection method, to confirm the efficiency of EV miRNA analysis after filter-based isolation. A MB is a hairpin-shaped molecular probe containing a fluorophore and quencher at its 3 ' and 5 ' ends, respectively ${ }^{29-31}$. In the presence of the target sequence, the MB hairpin structure is released, following which it specifically hybridizes with the target sequence and produces a strong fluorescent signal. The MBs used to target miRNA in this study are summarized in Supplementary Table S2. miR-21, miR-27a, and miR-375 in EVs were targeted to detect cancer because they are known regulators of carcinogenesis with aberrant expression $^{32-35}$. Differential miRNA expression is closely associated with various diseases ${ }^{36,37}$, and miR-21 and miR-27a are known to be upregulated, while miR-375 is downregulated, in $\mathrm{GC}^{32,33}$, hepatocellular carcinoma $(\mathrm{HCC})^{35}$ and esophageal adenocarcinoma ${ }^{34}$.

To test the efficiency of synthetic target miRNA detection with our MBs, we first analyzed the limit of detection (LOD), cross-reactivity, and multiplex detection of miRNAs, which are explained in detail in Fig. S4. To directly detect miRNA in filtered EVs, we first optimized the dilution and hybridization buffer through simultaneous one-step EV lysis and hybridization with MBs (Fig. S5). RIPA buffer and a Triton X-100 solution were selected for testing because they are transparent and the most commonly used EV lysis buffers. MB21 was premixed with the lysis solution for one-step detection, and a DPBS solution was used for the control group. After EV lysis and MB hybridization, their efficiencies were assessed based on fluorescence intensity, and we determined that the Triton X-100 solution was a better buffer for this purpose than RIPA buffer as the Triton X-100 solution yielded a higher $\mathrm{S} / \mathrm{B}$ ratio.

The $S / B$ ratios for the detection of miR-21, $-27 \mathrm{a}$, and -375 in a $5 \mathrm{~mL}$ sample were $\sim 1.26-, 1.3-$, and 1.13-fold higher, respectively, using the exofilter procedure 
versus centrifuge-based isolation $(p<0.05, p<0.01$, and $p<0.001$, respectively; Fig. 3d). Our approach was thus more efficient than conventional centrifuge-based isolation. For the multiplexed detection of miRNAs in EVs from CM, we coincubated a combination of MB21, MB27a, and MB375 with isolated EVs (Fig. 3e). The S/B ratios for the detection of three miRNAs (miR-21 + miR$27 \mathrm{a}+$ miR-375) in EVs using MB21 were almost the same as that for individual miRNA (miR-21) detection. Similarly, the S/B ratios for the detection of both miR-27a and miR-375 in EVs using MB27a and MB375, respectively, were 23.6 and $2.6 \%$ lower than those for individual miR$27 \mathrm{a}$ and miR-375 detection, respectively. Thus, multiple miRNAs in EVs were successfully detected in filtered EVbioadhesive nanoaggregates.

\section{Cell type-dependent expression of miRNA in EVs}

To further confirm the cell type-dependent expression patterns of miRNAs, we measured EVs secreted in the CM of various GC cell lines (AGS, MKN-74, and Hs746T cells) and an esophageal adenocarcinoma cell line (SKGT4 cells) as representative tumor cells and HEK293T and human fibroblast cells as representative normal cells. We performed the exofilter procedure and compared the results to those determined by CKs (Fig. 4a).

The $\mathrm{S} / \mathrm{B}$ ratio determined through the exofilter procedure was much higher than that determined with CK \#1 and $C K \# 2$. In particular, the S/B ratio for miR21 detection in HEK293T cells using the exofilter procedure was 1.44- and 2.3-fold higher than those using CK \#1 and CK \#2, respectively (Fig. 4b). Similarly, the S/B ratio for the detection of miR27a was 1.44- and 2.3-fold higher than those for its detection using CK \#1 and \#2, respectively, while the S/B ratio for the detection of miR-375 was 1.42 and 2.1-fold higher than those for its detection using CK $\# 1$ and $\# 2$, respectively. However, there were no significant differences in the S/B ratios for miR-21, -27a, and -375 detection between tumor and normal cell lines (Fig. 4b). However, EVs and miRNA expression levels in EVs differed across cell lines. HEK293T cells had higher S/B ratios for the detection of miR-21, $-27 \mathrm{a}$, and -375 than the other cancer cell lines, so we suspected that HEK293T cells simply released more EVs than the other cancer cells. This speculation was confirmed by measuring the concentration of the extracted total protein and analyzing the exosome surface markers CD9 and CD63 (Fig. S6).

For a more appropriate comparison, we analyzed the ratio between upregulated (up) and downregulated (down) expression. The expression ratios of miR-21 (up)/ miR-375 (down) and miR-27 (up)/miR-375 (down) were higher in the tumor cell lines than in the normal cell lines (Fig. 4c). In laryngeal squamous cell carcinoma (SCC) cells, the miR-21/miR-375 expression ratio was used as a combined biomarker by dividing the expression levels of upregulated miRNAs by those of downregulated miR$\mathrm{NAs}^{38}$. The miR-21/miR-375 and miR-27a/miR-375 expression ratios were significantly different between tumor and normal cell lines $(p<0.001)$. We performed RT-qPCR to confirm that these results were similar to those obtained from MBs (Fig. S7). Thus, our approach has the potential to diagnose cancer without the complicated steps required for RT-qPCR.

\section{Analysis of miRNA expression in EVs from patient blood plasma}

To verify the clinical utility of our approach, we performed exofilter-based miRNA detection in EVs from human blood plasma samples. We isolated EVs from the blood plasma of GC $(n=3)$ and HCC patients $(n=3)$ and healthy donors $(n=5)$ using our polyphenol-assisted method (Fig. 5a). We confirmed the miR-21, -27a, and -375 expression profiles using the online-accessible Gene Expression Omnibus (GEO) database and prepared a heatmap of those expression profiles in GC and HCC patients for comparison with those in healthy donors (Fig. S8A, C). The results of previous studies ${ }^{32,39}$ and those of our miRNA heatmap analysis (GSE23739, GSE54397, GSE21362, GSE22059) were consistent in that increased miR-21 and miR-27a expression and decreased miR-375 expression were detected in both GC and HCC samples compared with normal samples. Using the GEO database, we found that miRNA-21 and miR27a expression was significantly increased, whereas miR375 expression was significantly decreased, in GC patient samples (Fig. S8A, B $p<0.01, p<0.001$, and $p<0.0001$, respectively). In $\mathrm{HCC}$ patient samples, miR-21 was significantly increased, and miR-375 was significantly decreased; there was no significant difference in miR-27a expression (Fig. S8C, D, $p<0.0001$ ). Our data indicate that miR-21 expression was significantly higher in GC ( $p$ $<0.001)$ and HCC $(p<0.01)$ patients, while miR-375 expression was lower in HCC $(p<0.001)$ patients than in healthy donors (Fig. 5b). To quantify miRNA expression in GC plasma samples, we measured the relative levels of miR-21, miR-27a, and miR-375 using RT-qPCR (Fig. 5d). The miR-21/miR-375 and miR-27a/miR-375 expression ratios in plasma from cancer patients and healthy donors were significantly different. The miR-21/miR-375 expression ratio was higher in GC $(p<0.05)$ and $\mathrm{HCC}(p$ $<0.001)$ patients, and the miR-27a/miR-375 expression ratio was higher in HCC $(p<0.01)$ patients than in healthy donors (Fig. 5c, e). Although cancer patient samples showed significantly higher miR-21/miR-375 and $\mathrm{miR}-27 \mathrm{a} / \mathrm{miR}-375$ expression ratios, a large cohort should be analyzed in future studies to identify applicable cancer biomarkers for use in noninvasive liquid biopsies. 


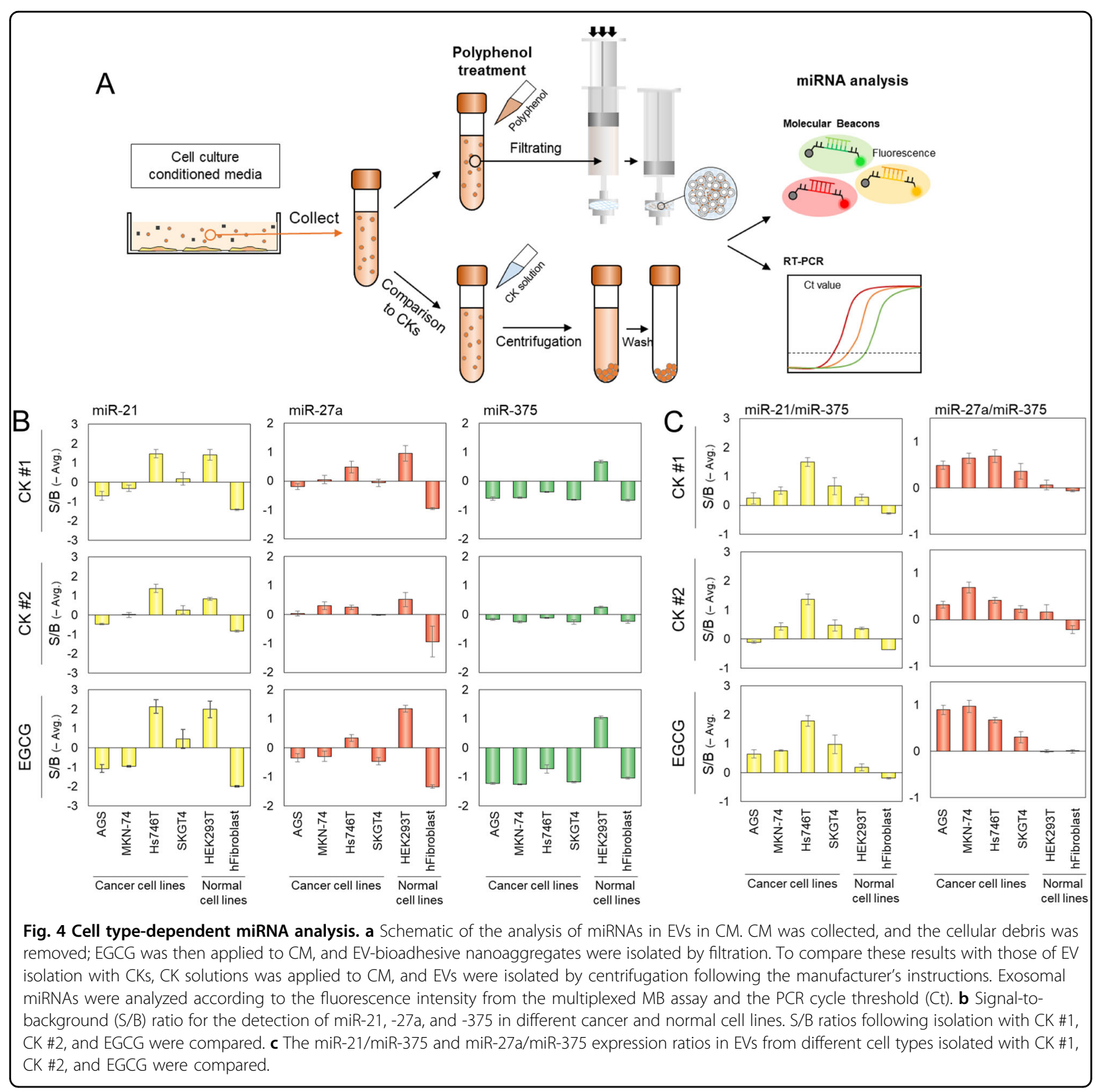

\section{Conclusions}

We designed a novel, cost-effective method to isolate nanosized EVs by using polyphenolic molecules. Our study demonstrates a potential translatable, efficient, and easy-to-use strategy with applications in molecular diagnostics of clinical samples. This study further highlights the use of a bioadhesive-mediated clinical sample preparation method that can be used effectively for sample isolation and enrichment for exosome-based molecular diagnostics. Analysis of exosomal miRNA in blood plasma from GC and HCC patients revealed higher miR-21/miR375 and miR-27a/miR-375 expression ratios, suggesting that our method can be used for exosome-based molecular diagnosis for personalized treatment and to monitor cancer.

\section{Acknowledgements}

This research was funded by the Bio \& Medical Technology Development Program of the National Research Foundation (NRF) funded by the Korean government (MSIP\&MOHW) [NRF-2015M3A9B3028685] and the Ministry of Science, ICT and Future Planning [2016M3A9B4915823]. This research was supported by the Korea Health Technology R\&D Project through the Korea Health Industry Development Institute (KHIDI) funded by the Ministry of Health \& Welfare [HI14C1324] and the KAIST Future Systems Healthcare Project from the Ministry of Science and ICT [KAISTHEALTHCARE42]. 


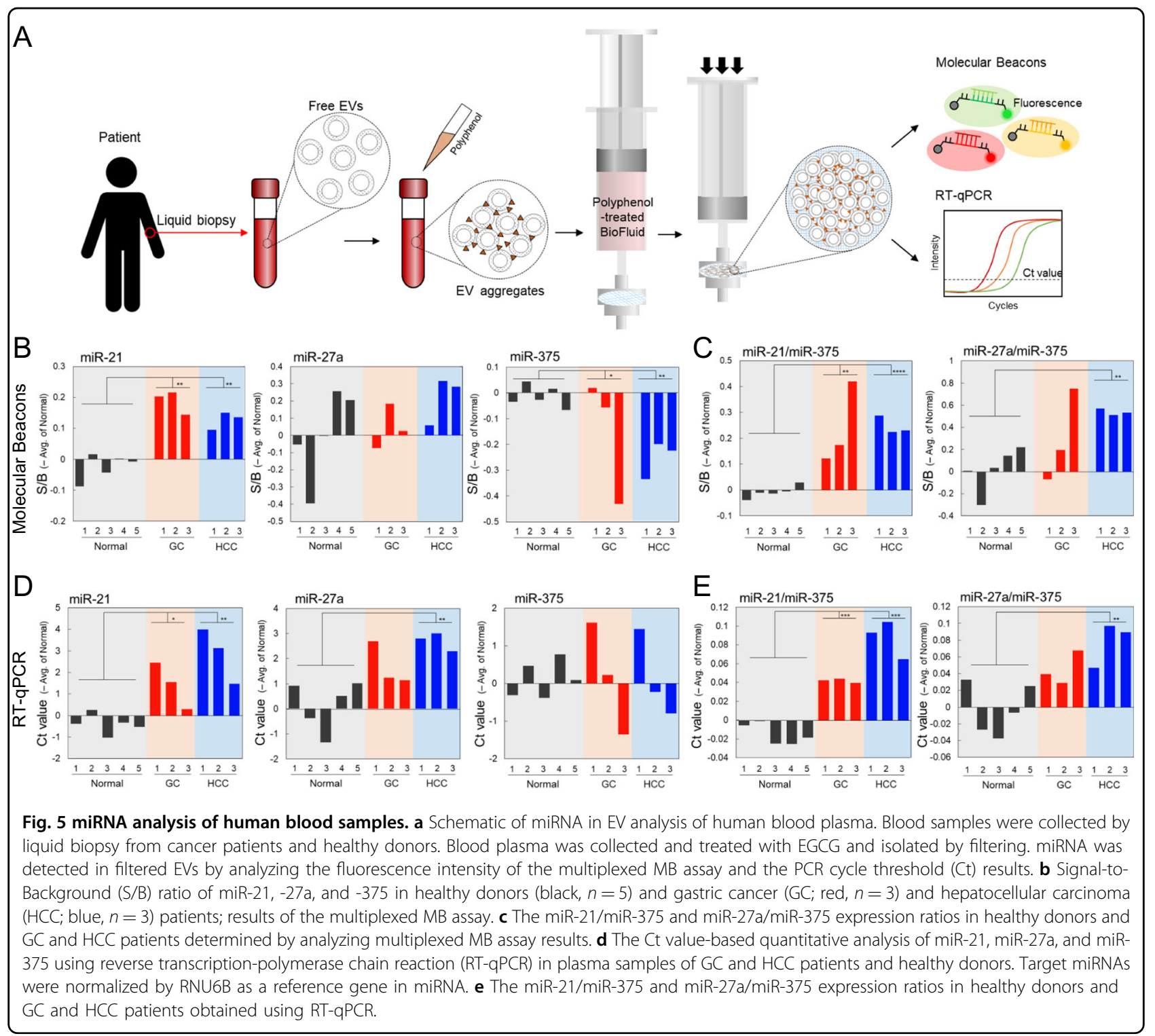

\section{Author details}

'Department of Bio and Brain Engineering, Korea Advanced Institute of Science and Technology (KAIST), Daejeon 34141, South Korea. ${ }^{2}$ Department of Mechanical Engineering, Korea Advanced Institute of Science and Technology (KAIST), Daejeon 34141, South Korea. ${ }^{3}$ Department of Surgery, Yonsei

University College of Medicine, Severance Hospital, Seoul 03722, South Korea. ${ }^{4}$ Department of Hepatobiliary and Pancreatic Surgery, Yonsei University College of Medicine, Severance Hospital, Seoul 03722, South Korea. ${ }^{5}$ Department of Chemistry, Korea Advanced Institute of Science and Technology (KAIST), Daejeon 34141, South Korea. ${ }^{6}$ KAIST Institute for Health Science and Technology, Daejeon 34141, South Korea

\section{Conflict of interest}

The authors declare that they have no conflict of interest.

\section{Publisher's note}

Springer Nature remains neutral with regard to jurisdictional claims in published maps and institutional affiliations.
Supplementary information is available for this paper at https://doi.org/ 10.1038/s41427-019-0184-0.

Received: 1 July 2019 Revised: 2 October 2019 Accepted: 6 October 2019. Published online: 13 December 2019

\section{References}

1. Shao, H. et al. New technologies for analysis of extracellular vesicles. Chem. Rev. https://doi.org/10.1021/acs.chemrev.7b00534 (2018).

2. Rani, S. \& Ritter, T. The exosome-a naturally secreted nanoparticle and its application to wound healing. Adv. Mater. 28, 5542-5552 (2016).

3. Wang, Y., Sun, S., Zhang, Z. \& Shi, D. Nanomaterials for cancer precision medicine. Adv. Mater. 30, e1705660 (2018).

4. Tay, H. M. et al. Rapid purification of sub-micrometer particles for enhanced drug release and microvesicles isolation. Npg Asia Mater. 9, e434. https://doi. org/10.1038/am.2017.175 (2017).

5. Whiteside, T. L. Tumor-derived exosomes and their role in cancer progression. Adv. Clin. Chem. 74, 103-141 (2016). 
6. Cesi, G., Walbrecq, G., Margue, C. \& Kreis, S. Transferring intercellular signals and traits between cancer cells: extracellular vesicles as "homing pigeons". Cell Commun. Signal 14, 13 (2016).

7. Shao, $\mathrm{H}$. et al. Chip-based analysis of exosomal mRNA mediating drug resistance in glioblastoma. Nat. Commun. 6, 6999 (2015).

8. Woo, H. K. et al. Exodisc for rapid, size-selective, and efficient isolation and analysis of nanoscale extracellular vesicles from biological samples. ACS Nano. 11, 1360-1370 (2017)

9. Zhao, Z., Yang, Y., Zeng, Y. \& He, M. A microfluidic ExoSearch chip for multiplexed exosome detection towards blood-based ovarian cancer diagnosis. Lab. Chip. 16, 489-496 (2016).

10. Weng, $Y$. et al. Effective isolation of exosomes with polyethylene glycol from cell culture supernatant for in-depth proteome profiling. Analyst 141, 4640-4646 (2016).

11. Im, H. et al. Label-free detection and molecular profiling of exosomes with a nano-plasmonic sensor. Nat. Biotechnol. 32, 490-495 (2014).

12. Contreras-Naranjo, J. C., Wu, H. J. \& Ugaz, V. M. Microfluidics for exosome isolation and analysis: enabling liquid biopsy for personalized medicine. Lab. Chip. 17, 3558-3577 (2017)

13. Wan, Y. et al. Rapid magnetic isolation of extracellular vesicles via lipidbased nanoprobes. Nat. Biomed. Eng. https://doi.org/10.1038/s41551-0170058 (2017).

14. Liang, K. et al. Nanoplasmonic quantification of tumor-derived extracellular vesicles in plasma microsamples for diagnosis and treatment monitoring. Nat. Biomed. Eng. https://doi.org/10.1038/s41551-016-0021 (2017).

15. Ibsen, S. D. et al. Rapid isolation and detection of exosomes and associated biomarkers from plasma. ACS Nano. 11, 6641-6651 (2017).

16. Lewis, J. M. et al. Integrated analysis of exosomal protein biomarkers on alternating current electrokinetic chips enables rapid detection of pancreatic cancer in patient blood. ACS Nano. 12, 3311-3320 (2018).

17. Yasui, T. et al. Unveiling massive numbers of cancer-related urinary-microRNA candidates via nanowires. Sci Adv. e1701133. https:/doi.org/10.1126/ sciadv.1701133 (2017).

18. Gao, Z. F. et al. Naked-eye point-of-care testing platform based on a pHresponsive superwetting surface: toward the non-invasive detection of glucose. Npg Asia Mater. 10, 177-189 (2018).

19. Sileika, T. S., Barrett, D. G., Zhang, R., Lau, K. H. \& Messersmith, P. B. Colorless multifunctional coatings inspired by polyphenols found in tea, chocolate, and wine. Angew. Chem. Int Ed. Engl. 52, 10766-10770 (2013).

20. Barrett, D. G., Sileika, T. S. \& Messersmith, P. B. Molecular diversity in phenolic and polyphenolic precursors of tannin-inspired nanocoatings. Chem. Commun. (Camb.) 50, 7265-7268 (2014).

21. Uekusa, Y., Kamihira, M. \& Nakayama, T. Dynamic behavior of tea catechins interacting with lipid membranes as determined by NMR spectroscopy. J. Agric Food Chem. 55, 9986-9992 (2007).
22. Xiao, J. \& Kai, G. A review of dietary polyphenol-plasma protein interactions: characterization, influence on the bioactivity, and structure-affinity relationship. Crit. Rev. Food Sci. Nutr. 52, 85-101 (2012).

23. Liang, K., Chung, J. E., Gao, S. J., Yongvongsoontorn, N. \& Kurisawa, M. Highly augmented drug loading and stability of micellar nanocomplexes composed of doxorubicin and poly(ethylene glycol)-green tea catechin conjugate for cancer therapy. Adv. Mater. 30, 1706963. https://doi.org/ 10.1002/adma.201706963 (2018)

24. Uekusa, Y. et al. Interaction of epicatechin gallate with phospholipid membranes as revealed by solid-state NMR spectroscopy. Biochim Biophys. Acta 1808, 1654-1660 (2011).

25. Shavandi, A. et al. Polyphenol uses in biomaterials engineering. Biomaterials 167, 91-106 (2018)

26. Pires, F. et al. On the role of epigallocatechin-3-gallate in protecting phospholipid molecules against UV irradiation. Colloids Surf. B Biointerfaces 173, 312-319 (2019).

27. Li, P., Kaslan, M., Lee, S. H., Yao, J. \& Gao, Z. Progress in Exosome Isolation Techniques. Theranostics 7, 789-804 (2017).

28. Deregibus, M. C. et al. Charge-based precipitation of extracellular vesicles. Int J. Mol. Med 38, 1359-1366 (2016).

29. Baker, M. B., Bao, G. \& Searles, C. D. In vitro quantification of specific microRNA using molecular beacons. Nucleic Acids Res 40, e13 (2012).

30. Lee, J. H., Kim, J. A., Jeong, S. \& Rhee, W. J. Simultaneous and multiplexed detection of exosome microRNAs using molecular beacons. Biosens. Bioelectron. 86, 202-210 (2016)

31. Lee, J. H., Kim, J. A., Kwon, M. H., Kang, J. Y. \& Rhee, W. J. In situ single step detection of exosome microRNA using molecular beacon. Biomaterials 54, 116-125 (2015).

32. Ishiguro, H., Kimura, M. \& Takeyama, H. Role of microRNAs in gastric cancer. World J. Gastroentero 20, 5694-5699 (2014).

33. Lai, $X$. et al. A microRNA signature in circulating exosomes is superior to exosomal glypican-1 levels for diagnosing pancreatic cancer. Cancer Lett. 393 86-93 (2017).

34. Gu, J., Wang, Y. \& Wu, X. F. MicroRNA in the pathogenesis and prognosis of esophageal cancer. Curr. Pharm. Des. 19, 1292-1300 (2013).

35. Gong, J., He, X. X. \& Tian, A. Emerging role of microRNA in hepatocellular carcinoma (Review). Oncol. Lett. 9, 1027-1033 (2015).

36. Jiang, $X$. et al. miR-22 has a potent anti-tumour role with therapeutic potential in acute myeloid leukaemia. Nat. Commun. 7, 11452. https://doi.org/10.1038/ ncomms11452 (2016).

37. Lee, S. E. et al. Integrated miRNA and mRNA expression profiling in response to eriodictyol in human endothelial cells. Biochip J. 11, 188-195 (2017).

38. Hu, A. et al. MiR-21/miR-375 ratio is an independent prognostic factor in patients with laryngeal squamous cell carcinoma. Am. J. Cancer Res. 5, 1775-1785 (2015).

39. Wu, X., Tan, X. \& Fu, S. W. May circulating microRNAs be gastric cancer diagnostic biomarkers? J. Cancer 6, 1206-1213 (2015). 